\title{
Technical note: The development of a reliable 5-point gait scoring system for use in dairy goats
}

\author{
L. E. Deeming, ${ }^{*} \dagger$ N. J. Beausoleil,† K. J. Stafford, $\dagger$ J. R. Webster, ${ }^{*}$ and G. Zobel ${ }^{* 1}$ \\ *AgResearch Ltd., Ruakura Research Centre, 10 Bisley Road, Hamilton 3214, New Zealand \\ †Institute of Veterinary, Animal and Biomedical Sciences, Massey University, Palmerston North 4410, New Zealand
}

\begin{abstract}
Numerical rating scales are frequently used in gait scoring systems as indicators of lameness in dairy animals. The gait scoring systems commonly used in dairy goats are based on 4-point scales that focus on detecting and judging the severity of a definite limp. An uneven gait, such as a shortened stride or not "tracking up," is arguably the precursor to the development of a limp; thus, identifying such changes in gait could provide opportunity for early treatment. The objectives of this study were (1) to develop a 5-point gait scoring system that included an "uneven gait" category and compare the distribution of gait scores generated using this system to scores generated using a 4 -point system, and (2) to determine whether this system could be reliably used. Forty-eight Saanen cross 2- and 3-yr-old lactating does were enrolled from a commercial dairy goat farm. Two observers carried out weekly live gait scoring sessions for 7 wk using the developed 5-point scoring system. The first 2 wk were used as training sessions (training sessions 1-2), with the subsequent 5 wk completed as gait assessments (assessments 1-5). In addition to training session 1 being lived scored, the goats were also videorecorded. This allowed observer 1 to re-score the session 4 times: twice using the developed 5-point system and twice using the previously used 4-point system. Comparisons of score distributions could then be made. Using the 4-point system, $81 \%$ of the goats were assigned score 1 (normal gait). Using the 5-point system, only $36 \%$ of the goats were assigned score 1 (normal gait), with $50 \%$ assigned score 2 (uneven gait). High levels of intra-observer reliability were achieved by observer 1 using both gait scoring systems [weighted kappa $\left(\kappa_{\mathrm{w}}\right)=$ 1.00: 4-point, $\kappa_{\mathrm{w}}=0.96$ : 5 -point]. At training session 1 (wk 1$)$, inter-observer reliability was only moderate $\left(\kappa_{\mathrm{w}}\right.$ $=0.54$ ), but this was improved during the subsequent training session $2\left(\kappa_{\mathrm{w}}=0.89\right)$. Inter-observer reliability
\end{abstract}

Received October 5, 2017.

Accepted January 8, 2018.

${ }^{1}$ Corresponding author: gosia.zobel@agresearch.co.nz was high among assessments 1 to $5\left(\kappa_{\mathrm{w}}=0.90-1.00\right)$. During the training sessions, sensitivity for gait scores 1 and 2 was 77 and $65 \%$ (training session 1) and 89 and $94 \%$ (training session 2), respectively. Sensitivity was high among assessments 1 to 5 (score 1: 83-100\%, score 2: 97-100\%). This highlights the likely reason why existing gait scoring systems for dairy goats do not include an "uneven gait" category, as distinguishing it from a normal gait was challenging without training. In conclusion, with training, a 5-point gait scoring system could be reliably used. The 5-point system was found to be more sensitive than the 4-point system, allowing for a potential precursor to lameness to be identified. Further work is needed to determine whether the score can be reliably used in an on-farm setting.

Key words: welfare, lame, uneven gait, limp

\section{Technical Note}

Lameness, a painful condition (Whay et al., 1997) that impedes a normal walking gait, is one of the most serious welfare issues faced by dairy animals (von Keyserlingk et al., 2009). As lameness compromises animal welfare (Whay et al., 2003), it is essential that the lameness status of dairy animals can be quickly and reliably identified to facilitate the prompt detection and treatment of lame animals.

Gait scoring systems, which use a numerical rating scale to rank an animal's walking ability, are commonly used as an indicator of lameness presence and severity. Systems assessing gait have been established for several species (sheep: Ley et al., 1989; chickens: Weeks et al., 2000; cows: Flower and Weary, 2006; goats: Anzuino et al., 2010; pigs: Nalon et al., 2014).

The 4-point gait scoring systems frequently used for dairy goats require a definite limp to be recognized (Hill et al., 1997; Anzuino et al., 2010; Muri et al., 2013) for an animal to be identified as lame. Gait scores are then assigned based on limp severity (Table 1). A limp can be defined as an altered gait due to reluctance to bear weight on the affected limb (Leach et al., 2009). This reluctance results in an uneven foot fall 
because a sound limb will be moved more quickly than the lame limb (Leach et al., 2010). With the exception of injuries, many cases of lameness develop over time (de Mol et al., 2013). Therefore, the development of an uneven gait could be a precursor to a limp developing. An uneven gait may be recognized as a shortening of stride, the animal not "tracking up" (i.e., the hind hoof not stepping into the placement of the front hoof) when walking, or as swinging of the affected leg inwards or outwards at each stride (van der Waaij et al., 2005; Haskell et al., 2006).

A 5-point gait scoring system is frequently used as an indicator of lameness in dairy cows (O'Callaghan et al., 2003; Espejo et al., 2006; Flower and Weary, 2006). The dairy cow 5-point scoring system includes an "uneven gait" category, which allows for discrimination of slight variation from a "normal gait," and therefore may facilitate earlier detection of developing lameness. Not including an "uneven gait" category in scoring systems such as the 4-point system often used in goats (Hill et al., 1997; Anzuino et al., 2010; Muri et al., 2013) may result in animals that have a slight variation from a normal gait being scored as "normal." These animals will only be detected once a definite limp has developed.

An uneven gait is not necessarily indicative of lameness. For example, conformation, posture, and udder fill of the animal may affect gait (Flower and Weary, 2009). However, using a gait scoring system that includes this category provides an opportunity to investigate the cause of the unevenness. Then, if deemed necessary, interventions such as remedial hoof trimming or veterinary treatment can be administered, potentially preventing deterioration of the condition (Leach et al., 2012).

Simplifying a gait scoring system by reducing the number of categories may improve inter-observer reliability and repeatability (Schlageter-Tello et al., 2014). This could explain why the previously used dairy goat gait scoring systems have fewer than 5 categories and often focus on identifying severe lameness. However, for cows, it is reported that with extra training, similar inter-observer reliability can be achieved using a 5-point system and a 4-point system (Brenninkmeyer et al., 2007). This suggests that the repeatability of a gait scoring system is determined not just by the sensitivity of the score, but also by the observers and their level of training and experience.

This study had 2 objectives: (1) to develop a 5-point gait scoring system for goats that includes a category for "uneven gait" with no limp, and to compare the distribution of gait scores generated using this system to scores generated using a 4-point system that focuses on identifying a limp; and (2) to determine whether the 5 -point system can be reliably used.
The study was conducted at the AgResearch Goat Research Facility (Hamilton, New Zealand) and was approved by the AgResearch Ltd. Animal Ethics Committee (13700). Forty-eight Saanen cross 2- and 3-yrold lactating does were enrolled in October 2016. The goats were housed singly or in pairs on rubber matting and shavings in the indoor facility as part of a larger feeding trial.

The same 2 observers carried out weekly gait scoring sessions for 7 consecutive weeks: the first 2 wh were training sessions, followed by 5 assessment sessions. All gait scoring sessions were conducted at approximately $1600 \mathrm{~h}$, following the afternoon milking, to reduce any effect of milk fill and udder distention on gait (Flower et al., 2006). Goats were assessed while walking from the milking parlor back to their pens on a combination of hard rubber matting and concrete flooring. They left the milking parlor and walked toward the observers, passed them laterally at a distance of 3 to $5 \mathrm{~m}$, and then continued away from the observers to their home pen. This allowed for at least 4 full strides of walk to be viewed. Efforts were made by the observers to keep an equal distance from the goats. However, due to the layout of the housing facility relative to the milking parlor, this was not always possible. Goats exited the parlor one at a time, enabling the observers to view and score each before another was allowed to exit. They exited in an indiscriminate order at each gait scoring session, which would have minimized the risk of observers becoming familiar with the order and recognizing individual goats.

In the first week, the 2 observers live scored the goats using the 5-point scale (see below) to evaluate reliability. This session was completed with the observers scoring independently, allowing an initial inter-observer reliability to be calculated. Inter-observer agreement was only moderate [weighted kappa $\left(\boldsymbol{\kappa}_{\mathrm{w}}\right)=0.54$; Table $2]$. The observers aimed to achieve almost perfect agreement (0.81-0.99; Viera and Garrett, 2005) before assessments could begin; therefore, further training was needed. Thus, training session 2 was completed, with the observers being able to discuss scores being assigned; this improved agreement $\left(\kappa_{\mathrm{w}}=0.89\right.$; Table 2$)$.

In wk 1, the goats were also video-recorded ( $\mathrm{n}=$ 42; 6 missed due to goats rushing; HC-V270, Panasonic Camcorder, Osaka, Japan) to allow comparison of the distribution of scores generated using the 4- and 5 -point systems. At the completion of the 7 -wk trial, observer 1 scored these video recordings 4 times: twice using the 5-point system and then twice using a 4-point system (Anzuino et al., 2010). Each scoring occurred 1 wk apart to minimize the risk of observer 1 being familiar with the goats and the order they appeared on the video. 
TECHNICAL NOTE: GAIT SCORING OF DAIRY GOATS

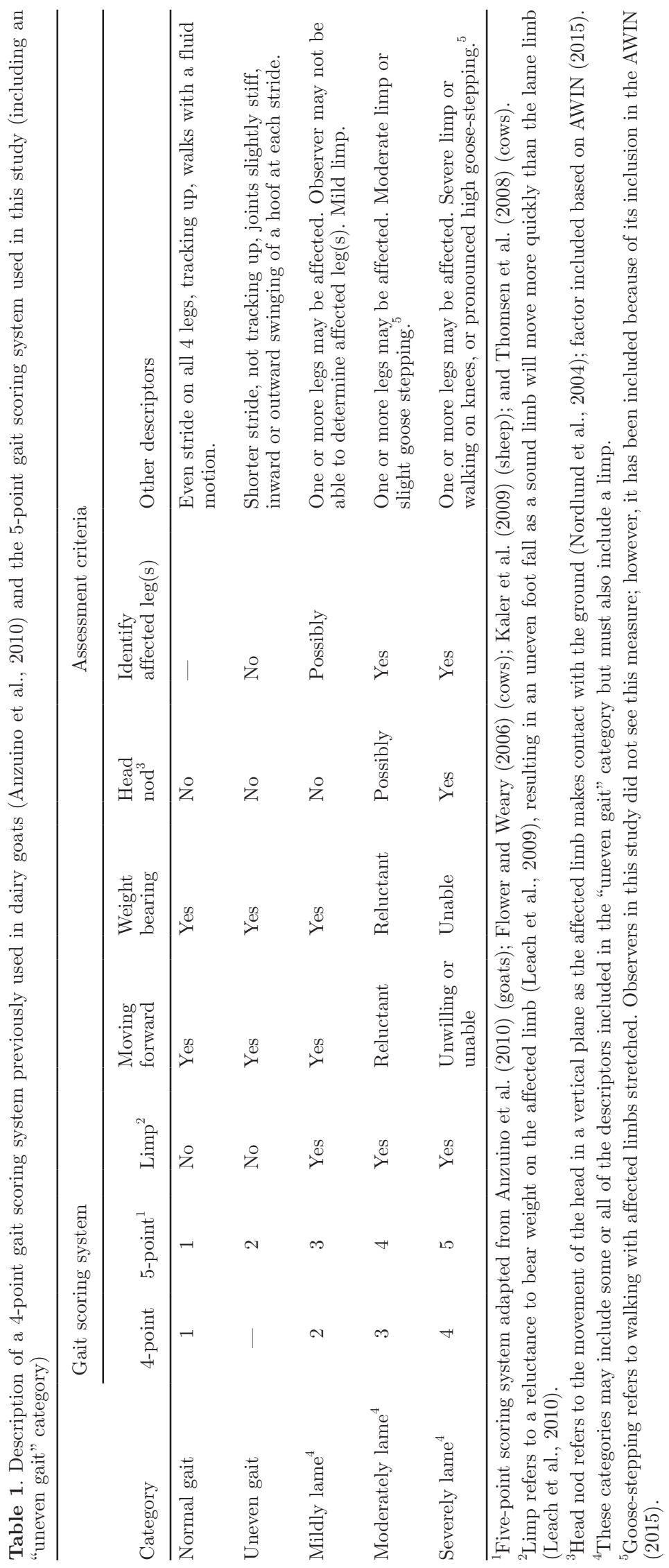


The PROC FREQ procedure of SAS (version 9.3, SAS Institute Inc., Cary, NC) was used to calculate $\kappa_{\mathrm{w}}$ and sensitivity (\%). This enabled inter-observer reliability at each gait scoring session to be evaluated, as well as intra-observer reliability of observer 1 when training session 1 video was re-scored. McNemar's test was performed to evaluate disagreements between the 2 observers within each training session and within each assessment session to establish whether there was consistent bias between the observers (e.g., one observer always scoring higher than the other).

The 5-point gait scoring system was developed using key descriptors (Table 1) from a previously used goat gait scoring system (Anzuino et al., 2010), combined with features from published scoring systems used for other species (e.g., cows: Flower and Weary, 2006; sheep: Kaler et al., 2009). These features include the quality of the gait, such as whether it is normal or uneven or a limp is present. They also include the animal's ability to move forward, its ability to bear weight, and the observer's ability to identify the affected leg(s). For moderate and severe lameness, features such as head nodding, "goose stepping" (walking with affected limbs stretched; AWIN, 2015), and walking on their knees have been included. The current study had a relatively small proportion of goats with moderate to severe lameness; therefore, the inclusion of these factors was done in accordance with the Animal Welfare Indicators (AWIN) welfare assessment protocol (AWIN, 2015), which focuses entirely on the identification of severe lameness.

High levels of intra-observer reliability were achieved by observer 1 using the developed 5-point gait scoring system and the previously used 4-point system $(\kappa \mathrm{w}=$ $0.96,5$-point; $\mathrm{kW}=1.00,4$-point). Using the 4-point system, the majority of the goats $(34 / 42,81 \%$; average of the 2 re-scores) were assigned score 1 (normal gait). However, using the 5-point scoring system, only 15 of 42 goats $(36 \%)$ were assigned score 1 , and 21 of 42 goats (50\%) were assigned score 2 (uneven gait; Figure 1). The difference in the distribution of the assigned gait scores when using the 2 systems indicates that several goats did not have a definite limp but also did not have a normal gait. When using the 4-point system, these "in-between" goats must be assigned a score 1 (normal gait). It should also be noted that 2 of the goats $(5 \%)$ scored as having a definite limp using the 4-point system were scored as having an uneven gait when the 5-point system was used. The observer considered these goats to be toward the higher end of the "uneven gait" category. Therefore, when using the 4 -point system, it was considered more appropriate to assign a "mild lameness" category, rather than "normal gait."

Study goats that were assigned a gait score of 2 (5-point scale; "uneven gait") or above at any of the assessments were investigated and promptly treated by a veterinarian if necessary. Therefore, it was not possible to monitor lameness progression across the 7-wk period. Prompt treatment also reduced the possibility of observers being able to recognize individual goats by their gait score. Observers could not assume that a high gait score at one gait scoring session would result in a high gait score at the subsequent gait scoring session.

Nearly half of the goats $(19 / 42 ; 44 \%)$ characterized as having a normal gait using the 4-point system were recognized as having an uneven gait using the 5-point system. Although this prevalence appears high, other

Table 2. Interobserver reliability between scores assigned by 2 observers for weekly gait scoring sessions for a period of 7 wk ( $\mathrm{n}=48$ goats)

\begin{tabular}{|c|c|c|c|c|c|c|c|}
\hline \multirow[b]{2}{*}{$\mathrm{Wk}^{1}$} & \multirow[b]{2}{*}{ Agreements } & \multicolumn{3}{|c|}{ Disagreements } & \multirow[b]{2}{*}{$\begin{array}{l}\text { Missed } \\
\text { scores }^{2}\end{array}$} & \multirow[b]{2}{*}{$\begin{array}{l}\text { McNemar's } \\
\text { test } P \text {-value }{ }^{3}\end{array}$} & \multirow[b]{2}{*}{$\begin{array}{l}\text { Weighted } \kappa \\
(95 \% \mathrm{CI})^{4}\end{array}$} \\
\hline & & $\begin{array}{c}\text { Observer } 1 \\
<\text { Observer } 2^{5}\end{array}$ & $\begin{array}{c}\text { Observer } 1 \\
>\text { Observer } 2^{6}\end{array}$ & Total & & & \\
\hline 1 & 28 & 8 & 6 & 14 & 6 & 0.59 & $0.54(0.34-0.74)$ \\
\hline 2 & 44 & 2 & 1 & 3 & 1 & 0.56 & $0.89(0.77-1.00)$ \\
\hline 3 & 41 & 0 & 2 & 2 & 5 & 0.10 & $0.90(0.76-1.00)$ \\
\hline 4 & 47 & 0 & 1 & 1 & 0 & 0.24 & $0.97(0.91-1.00)$ \\
\hline 5 & 41 & 0 & 0 & 0 & 7 & NA & $1.00(1.00-1.00)$ \\
\hline 6 & 47 & 1 & 0 & 1 & 0 & 0.24 & $0.97(0.90-1.00)$ \\
\hline 7 & 48 & 0 & 0 & 0 & 0 & NA & $1.00(1.00-1.00)$ \\
\hline
\end{tabular}

${ }^{1}$ The first 2 wk were used as training sessions, and the remaining weeks were used to assess interobserver reliability.

${ }^{2}$ Missed scores occurred when goats ran, which resulted in observers not being able to assign an accurate gait score.

${ }^{3}$ McNemar's test $P>0.10$ indicative of no consistent bias between observers within each assessment. NA $=$ McNemar's test cannot be performed because disagreements were 0 .

${ }^{4}$ Weighted $\kappa$ closest to 1.0 indicative of high levels of interobserver reliability.

${ }^{5}$ Disagreements when observer 1 assigned a gait score 1 point lower than observer 2 (the difference between observers was never greater than 1 point).

${ }^{6}$ Disagreements when observer 1 assigned a gait score 1 point higher than observer 2 (the difference between observers was never greater than 1 point). 
studies have suggested that an uneven gait can be very common in dairy animals. For example, of 183 dairy cows, $93 \%$ presented with the mildest lameness, considered equivalent to the "uneven gait" category in the 5-point system (Thomas et al., 2015). This highlights the potential lack of discrimination of low levels of lameness when fewer categories are included in a gait scoring system. It is important that the system enables precursors of an obvious limp to be detected, as these animals should be targeted for treatment, rather than waiting until the lameness becomes more severe (Nalon et al., 2014; Thomas et al., 2015).

The 5-point system frequently used to assess gait in cows includes variables other than limping. For instance, the "uneven gait" category uses the presence of an arched back while walking as a criterion to assign the cow to this category (Thomsen et al., 2008). In the present study, back arching did not become obvious until goats were moderately lame (score 4 on the 5-point scale). Indeed, when goats presented with an uneven gait, the observers viewed no other physical changes besides the slight deviation from normal walking. This extra challenge in identifying these goats may help to explain why the scoring systems previously used for dairy goats do not include the "uneven gait" category.

Several different gait scoring systems have been used as indicators of lameness in small ruminants. Similar to our findings for goats, 4-point scoring systems developed for use in sheep are not sensitive enough to detect lower degrees of lameness (Angell et al., 2015). Although 5-point scoring systems have also been developed for use in sheep, the categories are not well defined. They either use subjective descriptors, such as "obvious lameness" (Welsh et al., 1993), or do not give full descriptions of the categories used (Ley et al., 1989), making reproducibility difficult. Interestingly, a more detailed (7-point) scale including categories to detect an uneven gait was developed and reliably used in sheep (Kaler et al., 2009). In that study, the 3 observers were already familiar with gait scoring of sheep and received one training session using 10 video clips. Although they were able to identify the sheep with uneven gait, it should be noted that this was done entirely from recorded video clips; these authors did not test the scoring system in a live, on-farm setting.

In contrast, simplification of scoring by the use of a 4-point scale, or to a greater degree, the binary approach (i.e., severely lame or not lame) used in the AWIN protocol, may allow scoring to be achieved readily on farm with less training (AWIN, 2015). A binary score is reported to be used in the AWIN protocol because of the challenges of gait scoring dairy goats, such as husbandry constraints and differences in management and resources at farms (AWIN, 2015). Although even binary systems may have consistency issues over time (Can et al., 2017), they may be the best option for large-scale on-farm work, because they allow the prevalence of severe lameness to be identified quickly

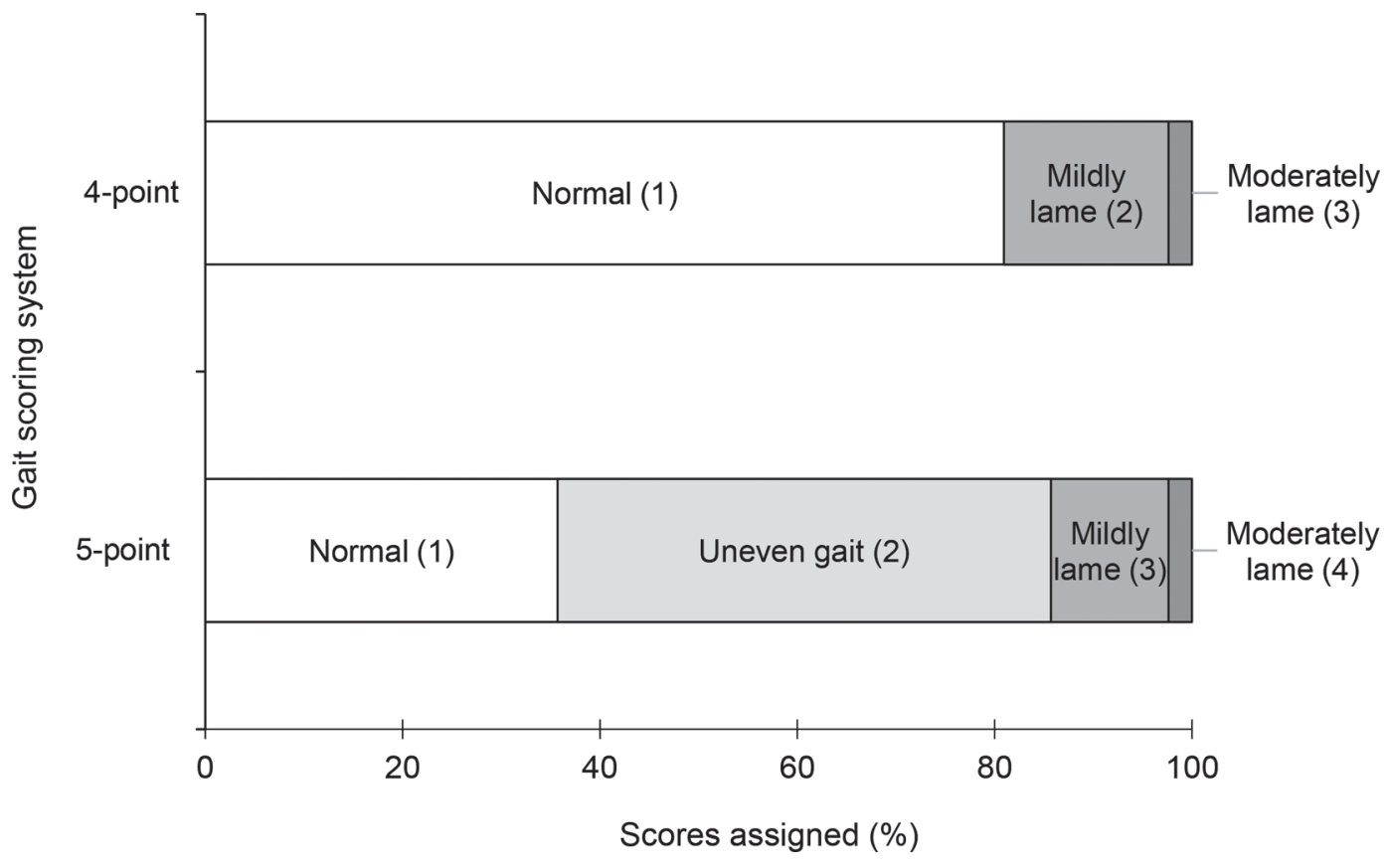

Figure 1. The distribution of gait scores assigned by observer 1 when scoring training session 1 (wk 1 ) videos using the 5-point gait scoring system (including "uneven gait" category) and the 4-point gait scoring system (no "uneven gait" category; Anzuino et al., 2010) (n = 42, 6 goats missed scoring). No goats were severely lame. Four-point score: $1=$ normal gait, $2=$ mildly lame, $3=$ moderately lame, $4=$ severely lame; five-point score: 1 = normal gait, $2=$ uneven gait, $3=$ mildly lame, $4=$ moderately lame, $5=$ severely lame. 
and easily during welfare assessments. The drawback of utilizing scoring systems with reduced categories is that, in large studies, the prevalence of less severe lameness may be underestimated.

Early detection and treatment are reported to reduce the prevalence of severe lameness and aid faster recovery (sheep: Kaler and Green, 2009; cows: Leach et al., 2012). In addition, lameness is known to negatively affect milk production (cows: Warnick et al., 2001; goats: Christodoulopoulos, 2009), fertility (cows: Melendez et al., 2003), and longevity (cows: Booth et al., 2004). Therefore, being able to detect an uneven gait, a potential indicator of early lameness, allows for further investigation and early treatment if necessary. This may reduce the negative effect on animal welfare and productivity.

Our second objective was to determine the reliability of the gait scoring system developed. Of the possible 240 scores from the weekly assessments (assessments 1-5 completed in wk 3-7), 227 observations were recorded. High inter-observer reliability was achieved using the 5-point scale for assessments 1 to $5\left(\kappa_{\mathrm{w}}=\right.$ 0.90 to 1.00; Table 2). We detected no difference in the disagreements of the 2 observers within any of the assessments (McNemar's $P$-value range: $0.10-0.24$; Table 2 ). This indicates no consistent bias between observers; that is, one observer did not consistently score higher or lower than the other observer.

Most (82\%) of the live scores assigned using the 5 -point scale over the 5 assessment sessions were scores 1 and 2. Scores 3, 4, and 5 comprised $13 \%$ of the total assigned scores, with $5 \%$ of goats missed. There were only 2 goats across the 5 assessments that each presented with severe lameness (score 5; 5-point system) at one of the assessments. The sensitivity between the 2 observers for scores 1 and 2 during the training sessions was 77 and $65 \%$ (training session 1) and 89 and $94 \%$ (training session 2), respectively. During assessments 1 to 5 , sensitivity was high (score 1: $83-100 \%$, score 2: $97-100 \%)$.

The low initial sensitivities during training highlights the likely reason that existing scoring systems do not include "uneven gait," because both observers found that distinguishing "uneven gait" from "normal gait" was challenging. Identifying an uneven gait, the intermediate category between normal gait and a definite limp, is also challenging in other small ruminants. For example, the greatest disagreement between observers scoring sheep was found between the "normal gait" category and the "slight abnormal gait" category (Kaler et al., 2009). Nonetheless, in the current study, following 2 training sessions, the observers could use the 5-point gait scoring system reliably.
The 13 missing scores were attributed to goats rushing after exiting the milking parlor. Although we attempted to reduce this behavior by having an assistant walk in front of the goats, it was still not possible to achieve a steady walking pace for all goats to assign an accurate gait score. This is pertinent because rushing was found, on at least one occasion, to almost entirely mask a score 4 (moderately lame; 5-point scale) goat. The ability to assign an accurate gait score is reduced if goats move faster than a walk. This is particularly relevant for animals with lower levels of lameness because subtler changes in gait are more difficult to detect as speed increases. Gait scores were only assigned by the 2 observers if the goats walked at a steady pace to ensure scoring was not biased by the speed of the goat. Difficulties in assigning accurate gait scores due to the speed that goats exit the milking parlor have previously been reported and resulted in a simple binary scoring system (lame/not lame) being used (Crosby-Durrani et al., 2016). Therefore, we suggest that controlling the speed of goats and ensuring goats walk at a steady consistent speed is essential to ensure accuracy of the 5 -point score. However, we acknowledge that this would not necessarily be feasible in all on-farm settings.

The observers developed the 5-point gait scoring system presented here and were therefore very familiar with the system before gait scoring was completed. However, further training sessions were required to improve inter-observer reliability. March et al. (2007) also found that considerable training, involving at least 5 farm visits and the scoring of between 200 and 300 live animals, was required to achieve high inter-observer repeatability when using a 5-point system to score gait in dairy cows. Less intensive training was required in the present study (2 training sessions comprising 42 and 47 animals, respectively). Although training can be time consuming, the result is a reliable 5-point gait scoring system. Nonetheless, it should be noted that the present study focused on a small number of animals in a controlled environment. Therefore, we caution that due to the challenges of gait scoring dairy goats, large-scale farm work needs to be completed to determine whether the 5-point gait scoring system presented here is applicable to on-farm settings and prevalence assessments.

In conclusion, we successfully developed a 5-point gait scoring system. After 2 training sessions, reliability was achieved between 2 observers scoring a small group of goats. Nearly half of the goats characterized as having a normal gait using a previously reported 4-point system were recognized as having an uneven gait using the developed 5-point system. Using a scoring system that enables the identification of an uneven gait could facilitate detection of early signs of lameness, allow- 
ing for early investigation and treatment if necessary. We encourage the testing of this 5-point gait scoring system in large, on-farm settings.

\section{ACKNOWLEDGMENTS}

Funding for the research and the student stipend was provided by the NZ Ministry of Business, Innovation and Employment (MBIE, Wellington, New Zealand), and the Dairy Goat Cooperative (NZ) Ltd. (DGC, Hamilton, New Zealand). Special thanks is given to the participating DGC farmer.

\section{REFERENCES}

Angell, J. W., P. J. Cripps, D. H. Grove-White, and J. S. Duncan. 2015. A practical tool for locomotion scoring in sheep: Reliability when used by veterinary surgeons and sheep farmers. Vet. Rec. 176:521-523.

Anzuino, K., N. J. Bell, K. J. Bazeley, and C. J. Nicol. 2010. Assessment of welfare on 24 commercial UK dairy goat farms based on direct observations. Vet. Rec. 167:774-780.

AWIN. 2015. Animal Welfare Indicators (AWIN) welfare assessment protocol for goats. https://doi.org/10.13130/AWIN_goats_2015.

Booth, C. J., L. Warnick, Y. Gröhn, D. Maizon, C. Guard, and D. Janssen. 2004. Effect of lameness on culling in dairy cows. J. Dairy Sci. 87:4115-4122.

Brenninkmeyer, C., S. Dippel, S. March, J. Brinkmann, C. Winckler, and U. Knierim. 2007. Reliability of a subjective lameness scoring system for dairy cows. Anim. Welf. 16:127-129.

Can, E., A. Vieira, M. Battini, S. Mattiello, and G. Stilwell. 2017. Consistency over time of animal-based welfare indicators as a further step for developing a welfare assessment monitoring scheme: The case of the Animal Welfare Indicators protocol for dairy goats. J. Dairy Sci. 100:9194-9204.

Christodoulopoulos, G. 2009. Foot lameness in dairy goats. Res. Vet. Sci. 86:281-284.

Crosby-Durrani, H. E., S. R. Clegg, E. R. Singer, J. W. Angell, N J. Evans, S. D. Carter, R. J. Blundell, and J. S. Duncan. 2016. Severe foot lesions in dairy goats associated with digital dermatitis treponemes. J. Comp. Pathol. 154:283-296.

de Mol, R. M., G. André, E. J. B. Bleumer, J. T. N. Van Der Werf, Y. De Haas, and C. G. Van Reenen. 2013. Applicability of day-to-day variation in behavior for the automated detection of lameness in dairy cows. J. Dairy Sci. 96:3703-3712.

Espejo, L. A., M. I. Endres, and J. A. Salfer. 2006. Prevalence of lameness in high-producing holstein cows housed in freestall barns in Minnesota. J. Dairy Sci. 89:3052-3058.

Flower, F. C., D. Sanderson, and D. M. Weary. 2006. Effects of milking on dairy cow gait. J. Dairy Sci. 89:2084-2089.

Flower, F. C., and D. M. Weary. 2006. Effect of hoof pathologies on subjective assessments of dairy cow gait. J. Dairy Sci. 89:139-146.

Flower, F. C., and D. M. Weary. 2009. Gait assessment in dairy cattle. Animal 3:87-95.

Haskell, M. J., L. Rennie, V. Bowell, M. Bell, and A. Lawrence. 2006. Housing system, milk production, and zero-grazing effects on lameness and leg injury in dairy cows. J. Dairy Sci. 89:4259-4266.

Hill, N. P., P. E. Murphy, A. J. Nelson, L. E. Mouttotou, L. E. Green, and K. L. Morgan. 1997. Lameness and foot lesions in adult British dairy goats. Vet. Rec. 141:412-416.

Kaler, J., and L. E. Green. 2009. Farmers' practices and factors associated with the prevalence of all lameness and lameness attributed to interdigital dermatitis and footrot in sheep flocks in England in 2004. Prev. Vet. Med. 92:52-59.

Kaler, J., G. J. Wassink, and L. E. Green. 2009. The inter- and intraobserver reliability of a locomotion scoring scale for sheep. Vet. J. 180:189-194
Leach, K. A., S. Dippel, J. Huber, S. March, C. Winckler, and H. R. Whay. 2009. Assessing lameness in cows kept in tie-stalls. J. Dairy Sci. 92:1567-1574.

Leach, K. A., D. A. Tisdall, N. J. Bell, D. C. J. Main, and L. E. Green. 2012. The effects of early treatment for hindlimb lameness in dairy cows on four commercial UK farms. Vet. J. 193:626-632.

Leach, K. A., H. R. Whay, C. M. Maggs, Z. E. Barker, E. S. Paul, A. K. Bell, and D. C. J. Main. 2010. Working towards a reduction in cattle lameness: 1 . Understanding barriers to lameness control on dairy farms. Res. Vet. Sci. 89:311-317.

Ley, S. J., A. Livingston, and A. E. Waterman. 1989. The effect of chronic clinical pain on thermal and mechanical thresholds in sheep. Pain 39:353-357.

March, S., J. Brinkmann, and C. Winkler. 2007. Effect of training on the inter-observer reliability of lameness scoring in dairy cattle. Anim. Welf. 16:131-133.

Melendez, P., J. Bartolome, L. F. Archbald, and A. Donovan. 2003. The association between lameness, ovarian cysts and fertility in lactating dairy cows. Theriogenology 59:927-937.

Muri, K., S. M. Stubsjøen, and P. S. Valle. 2013. Development and testing of an on-farm welfare assessment protocol for dairy goats. Anim. Welf. 22:385-400.

Nalon, E., D. Maes, S. Van Dongen, M. M. Van Riet, G. Janssens, S. Millet, and F. Tuyttens. 2014. Comparison of the inter-and intraobserver repeatability of three gait-scoring scales for sows. Animal 8:650-659.

Nordlund, K. V., N. B. Cook, and G. R. Oetzel. 2004. Investigation strategies for laminitis problem herds. J. Dairy Sci. 87:E27-E35.

O'Callaghan, K. A., P. J. Cripps, D. Y. Downham, and R. D. Murray. 2003. Subjective and objective assessment of pain and discomfort due to lameness in dairy cattle. Anim. Welf. 12:605-610.

Schlageter-Tello, A., E. A. M. Bokkers, P. W. G. Groot, T. Koerkamp, T. Van Hertem, S. Viazzi, C. E. B. Romanini, I. Halachmi, C. Bahr, D. Berckmans, and K. Lokhorst. 2014. Effect of merging levels of locomotion scores for dairy cows on intra- and interrater reliability and agreement. J. Dairy Sci. 97:5533-5542.

Thomas, H. J., G. G. Miguel-Pacheco, N. J. Bollard, S. C. Archer, N. J. Bell, C. Mason, O. J. R. Maxwell, J. G. Remnant, P. Sleeman, H. R. Whay, and J. N. Huxley. 2015. Evaluation of treatments for claw horn lesions in dairy cows in a randomized controlled trial. J. Dairy Sci. 98:4477-4486.

Thomsen, P. T., L. Munksgaard, and F. A. Tøgersen. 2008. Evaluation of a lameness scoring system for dairy cows. J. Dairy Sci. 91:119-126.

van Der Waaij, E. H., M. Holzhauer, E. Ellen, C. Kamphuis, and G. De Jong. 2005. Genetic parameters for claw disorders in Dutch dairy cattle and correlations with conformation traits. J. Dairy Sci. 88:3672-3678.

Viera, A. J., and J. M. Garrett. 2005. Understanding interobserver agreement: The kappa statistics. Fam. Med. 37:360-363.

von Keyserlingk, M. A. G., J. Rushen, A. M. De Passillé, and D. M. Weary. 2009. Invited review: The welfare of dairy cattle - Key concepts and the role of science. J. Dairy Sci. 92:4101-4111.

Warnick, L. D., D. Janssen, C. L. Guard, and Y. T. Gröhn. 2001. The effect of lameness on milk production in dairy cows. J. Dairy Sci. 84:1988-1997.

Weeks, C. A., T. D. Danbury, H. C. Davies, P. Hunt, and S. C. Kestin 2000. The behaviour of broiler chickens and its modification by lameness. Appl. Anim. Behav. Sci. 67:111-125.

Welsh, E. M., G. Gettinby, and A. M. Nolan. 1993. Comparison of a visual analogue scale and a numerical rating scale for assessment of lameness, using sheep as a model. Am. J. Vet. Res. 54:976-983.

Whay, H. R., D. C. J. Main, L. E. Green, and A. J. F. Webster. 2003. Assessment of the welfare of dairy cattle using animal-based measurements: Direct observations and investigation of farm records. Vet. Rec. 153:197-202

Whay, H. R., A. E. Waterman, and A. J. F. Webster. 1997. Associations between locomotion, claw lesions and nociceptive threshold in dairy heifers during the peri-partum period. Vet. J. 154:155-161. 